\title{
PARTITIONS INTO LARGE UNEQUAL PARTS FROM A GENERAL SEQUENCE
}

\author{
KEVIN JOHN FERGUSSON
}

(Received 18 June 1999; revised 28 January 2004)

Communicated by W. W. L. Chen

\begin{abstract}
An asymptotic estimate is obtained for the number of partitions of the positive integer $n$ into unequal parts coming from a sequence $u$, with each part greater than $m$, under suitable conditions on the sequence $u$. The estimate holds uniformly with respect to integers $m$ such that $0 \leq m \leq n^{1-\delta}$, as $n \rightarrow \infty$, where $\delta$ is a given real number, such that $0<\delta<1$.
\end{abstract}

2000 Mathematics subject classification: primary $11 \mathrm{P} 82$.

\section{Introduction}

Let $u=\left(u_{j}\right)_{j=1}^{\infty}$ be a given strictly increasing sequence of positive integers and let $m$ and $n$ be integers such that $0 \leq m<n / 2$. Define $q_{u}(m, n)$ to be the number of partitions of $n$ into unequal parts from $u$ with each part greater than $m$, that is, $q_{u}(m, n)$ is the number of ways of writing $n$ in the form

$$
n=u_{j_{1}}+u_{j_{2}}+\cdots+u_{j_{r}}
$$

where

$$
m<u_{j_{1}}<u_{j_{2}}<\cdots<u_{j_{r}},
$$

with $r$ arbitrary. The classical partition function $q(n)=q_{\mathbb{N}}(0, n)$ is the number of partitions of $n$ into distinct positive integers.

The aim of this paper is to study the asymptotic behaviour of $q_{u}(m, n)$ as $n \rightarrow \infty$ for a reasonably broad class of sequences $u$. The paper is based on the main result on general sequences in my Ph.D. thesis [5], to which I shall refer for some further details. In the paper [4] I give the detailed application to the sequence of $k$ th powers.

(c) 2006 Australian Mathematical Society 1446-7887/06 $\$ \mathrm{~A} 2.00+0.00$ 
Background history The first major work in asymptotic partition theory was the important 1918 paper of Hardy and Ramanujan [9] which presented their investigation of the classical partition functions $p(n)$ (the number of ordinary partitions of $n$ into positive integers which are not required to be distinct) and $q(n)$ as defined above. In particular, using the transformation theory of modular functions, they gave the result

$$
q(n) \sim \frac{\mathrm{e}^{\pi \sqrt{n / 3}}}{4.3^{1 / 4} \cdot n^{3 / 4}}, \quad \text { as } n \rightarrow \infty
$$

and a corresponding asymptotic estimate for $p(n)$ (estimates which had been found independently by Uspensky [16]). Their main result, however, was a theorem giving an asymptotic series expansion of $p(n)$ in terms of Bessel functions.

Later, in 1942 Hua [10] used a similar method to obtain an asymptotic series expansion of $q(n)$ in terms of Bessel Functions. In 1941 Ingham [12] developed a general Tauberian theorem for which he gave some applications, including, in particular, a simpler derivation of the above estimate of $q(n)$.

In 1954 Roth and Szekeres [14] proved a general theorem giving an asymptotic expansion for the number of partitions $q_{u}(0, n)$ of $n$ into distinct members of a general sequence $u$ satisfying appropriate conditions. The hypotheses on the sequence $u$ used by Roth and Szekeres were as follows:

$$
\begin{gathered}
\lim _{n \rightarrow \infty} \frac{\log u_{n}}{\log n}=s>0, \\
\inf \left((\log n)^{-1} \sum_{j=1}^{n}\left\|\alpha u_{j}\right\|^{2}\right) \rightarrow \infty \quad \text { as } n \rightarrow \infty,
\end{gathered}
$$

where the infimum is taken over $\alpha \in\left(\left(2 u_{n}\right)^{-1}, 1 / 2\right)$. Roth and Szekeres showed that Hypotheses (RS1) and (RS2) are satisfied by a wide range of sequences $u$, including sequences of the form $(P(j))$ and $\left(P\left(p_{j}\right)\right)$, where $p_{j}$ is the $j$ th prime number and $P$ is a suitable polynomial function in each case, and in particular including the sequences $u=\mathbb{N}$ and $\left(p_{j}\right)$, and the sequence of $k$ th powers $\left(j^{k}\right)$.

As a particular case of the main theorem of Roth and Szekeres we have the following asymptotic estimate of $q_{u}(0, n)$.

THEOREM A (Roth and Szekeres). Let $u=\left(u_{j}\right)$ be a sequence of positive integers which is strictly increasing for $j \geq j_{0}$ and which satisfies Hypotheses (RS1) and (RS2). For $q_{u}(0, n)$ as above and given $\delta>0$, we have, as $n \rightarrow \infty$,

$$
q_{u}(0, n)=\frac{1}{\sqrt{2 \pi A_{2}}} \exp \left(\sum_{j=1}^{\infty}\left(\frac{\sigma u_{j}}{1+\mathrm{e}^{\sigma u_{j}}}+\log \left(1+\mathrm{e}^{-\sigma u_{j}}\right)\right)\right)\left(1+O\left(n^{-(s+1)^{-1}+\delta}\right)\right),
$$

where $\sigma$ is determined from $n=\sum_{1}^{\infty} u_{j}\left(1+\mathrm{e}^{\sigma u_{j}}\right)^{-1}$. 
Their proof relied on writing $q_{u}(0, n)$ as the integral of a generating function and then splitting the range of integration into two parts-an interval around 0 , giving the main integral, and the remaining part, giving the supplementary integral. Their second hypothesis ensures that the supplementary integral is dominated by the main integral and they obtained their main term by estimating the main integral.

In 1960 Cassels [2] established conditions on a sequence $u$ ensuring the existence of a representation of every sufficiently large number $n$ as a sum of distinct members of the sequence $u$.

Freiman developed a method for partition problems which drew on the ideas of local limit theorems (see, for example, Freiman [6]) and recently Freiman and Pitman [7], using this method, proved the following result on the special case when $u=\mathbb{N}$, the sequence of all positive integers.

THEOREM B (Freiman and Pitman). For integers $m$ and $n$ such that $0 \leq m<n / 2$, let $\sigma$ be defined by $n=\sum_{m<j \leq n} j /\left(1+\mathrm{e}^{\sigma j}\right)$ and let $A_{2}=\sum_{m<j \leq n} j^{2} \mathrm{e}^{\sigma j} /\left(1+\mathrm{e}^{\sigma j}\right)^{2}$. Then as $n \rightarrow \infty$ we have

$$
q_{\mathrm{N}}(m, n)=\frac{1}{\sqrt{2 \pi A_{2}}} \mathrm{e}^{\sigma n} \prod_{m<j \leq n}\left(1+\mathrm{e}^{-\sigma j}\right)(1+E),
$$

where

$$
E=E(m, n)=O\left((\log n)^{9 / 2} \max \left(n^{-1 / 4},(m / n)^{1 / 2}\right)\right),
$$

uniformly with respect to $m$ such that $0 \leq m \leq K_{0} n / \log ^{9} n, K_{0}$ an effective positive constant.

Their analytical method is very similar to Roth and Szekeres but uses a different identity which is based on a lemma involving a finite product (see Lemma 1 below) rather than an infinite product.

Over the past few years Erdös, Nicolas and Szalay [3] have investigated the asymptotic behaviour of $q_{\mathbb{N}}(m, n)$, concentrating on the case when $m$ is relatively small (less than $n^{1 / 2}$ ).

The investigation of corresponding questions for ordinary partitions has gone ahead alongside, and sometimes ahead of, the work outlined above. Although the results obtained for partitions into distinct parts and for ordinary partitions are often parallel, some methods appear to work more easily for one than the other.

Postnikov [13] gives a good introduction to the whole field, including the use of probabilistic results.

This paper The main content of this paper is a theorem for a general sequence along the lines of Theorems A and B which is valid for a wide range of values of $m$ such that $0 \leq m<n / 2$. The work will draw heavily on the ideas of Roth and Szekeres [14] 
and Freiman and Pitman [7]. However the present problem involves added difficulties associated with replacing $q_{u}(0, n)$ by $q_{u}(m, n)$ in Theorem A (requiring careful estimation in terms of $m$ ) and also replacing $\mathbb{N}$ by a general sequence $u$ in Theorem B.

In Section 2, I give a probabilistic motivation for the main theorem based on the approach of Freiman and Pitman [7]. In Section 3, I introduce two hypotheses on the sequence $u$ to play the role of (RS1) and (RS2) and then in Section 4, I state the main theorem, outline its proof, and describe the remaining sections of this paper in which the proof will be given. Results in the paper will be numbered in order of occurrence (so that, for example, Lemma 1 precedes Theorem 2).

I am grateful for the assistance provided by my PhD supervisor Dr Jane Pitman and the members of the Number Theory Seminar at the University of Adelaide Department of Pure Mathematics. The work described here was assisted by a University of Adelaide Research Scholarship, for the period 16 March 1992 to 15 March 1995 during which most of the work was completed.

\section{Probabilistic motivation}

We commence with the following lemma.

LEMMA 1. Let $u=\left(u_{j}\right)_{j=1}^{\infty}$ be a strictly increasing sequence of positive integers and let $\sigma$ be a given real number. With $q_{u}(m, n)$ as above, we have

$$
q_{u}(m, n)=\mathrm{e}^{\sigma n} \prod_{m<u_{j} \leq n}\left(1+\mathrm{e}^{-\sigma u_{j}}\right) \int_{-1 / 2}^{1 / 2} \varphi(\alpha) \mathrm{e}^{-2 \pi i \alpha n} d \alpha,
$$

where

$$
\varphi(\alpha)=\prod_{m<u_{j} \leq n} \varphi_{j}(\alpha)
$$

and where

$$
\varphi_{j}(\alpha)=\frac{1}{1+\mathrm{e}^{-\sigma u_{j}}}+\frac{\mathrm{e}^{-\sigma u_{j}}}{1+\mathrm{e}^{-\sigma u_{j}}} \mathrm{e}\left(\alpha u_{j}\right)
$$

Proof. It is easily seen that $q_{u}(m, n)$ is the coefficient of $\mathrm{e}^{2 \pi i \alpha n}$ in the product

$$
\mathrm{e}^{\sigma n} \prod_{m<u_{j} \leq n}\left(1+\mathrm{e}^{-\sigma u_{j}} \mathrm{e}^{2 \pi i \alpha u_{j}}\right) \text {. }
$$

Using the fact that

$$
\int_{-1 / 2}^{1 / 2} \mathrm{e}^{2 \pi i \alpha k} d \alpha= \begin{cases}1 & \text { if } k=0 \\ 0 & \text { if } k \neq 0\end{cases}
$$

we see the result immediately. 
We now introduce some non-negative integer valued random variables in the context of the lemma. For such a random variable $X$, the function defined by $\varphi(\alpha)=\mathbb{E}\left(\mathrm{e}^{2 \pi i \alpha X}\right)$ will be called the characteristic function of $X$. (It is simply a rescaling of the classical characteristic function.)

As before, let $u$ be a strictly increasing sequence of positive integers, and as in the lemma let $\sigma$ be a given real number. For each positive integer $j$ define

$$
p_{1 j}=\frac{1}{1+\mathrm{e}^{-\sigma u_{j}}}, \quad p_{2 j}=\frac{\mathrm{e}^{-\sigma u_{j}}}{1+\mathrm{e}^{-\sigma u_{j}}}=1-p_{1 j} .
$$

We consider a sequence $\left(X_{j}\right)_{1}^{\infty}$ of independent non-negative integer-valued random variables such that

$$
\mathbb{P}\left(X_{j}=x\right)= \begin{cases}p_{1 j} & \text { for } x=0 \\ p_{2 j} & \text { for } x=u_{j}\end{cases}
$$

(Such a sequence exists by, for example, Ash [1, Section 5.11].) We note that $\varphi_{j}(\alpha)$ in (2.3) is the characteristic function of $X_{j}$.

Consider the random variable $Y=Y_{m, n}$ defined by

$$
Y=\sum_{m<u_{j} \leq n} X_{j}
$$

We have $\mathbb{E}(Y)=\sum_{m<u_{j} \leq n} p_{2 j} u_{j}$ and because of the independence of the $X_{j}$ 's we also have $\vee(Y)=\sum_{m<u_{j} \leq n} p_{1 j} p_{2 j} u_{j}^{2}$ and the characteristic function $\varphi$ of the random variable $Y$ is given by (2.2).

We denote the first and second cumulants of the random variable $Y$ by

$$
A_{1}=\sum_{m<u_{j} \leq n} f_{1}\left(u_{j}\right)=\sum_{m<u_{j} \leq n} \frac{u_{j}}{1+\mathrm{e}^{\sigma u_{j}}}
$$

and

$$
A_{2}=\sum_{m<u_{j} \leq n} f_{2}\left(u_{j}\right)=\sum_{m<u_{j} \leq n} \frac{u_{j}^{2} \mathrm{e}^{\sigma u_{j}}}{\left(1+\mathrm{e}^{\sigma u_{j}}\right)^{2}},
$$

where

$$
f_{1}(x)=\frac{x}{1+\mathrm{e}^{\sigma x}} \quad \text { and } \quad f_{2}(x)=\frac{x^{2} \mathrm{e}^{\sigma x}}{\left(1+\mathrm{e}^{\sigma x}\right)^{2}}
$$

We remark that

$$
\mathbb{E}(Y)=A_{1}, \quad \vee(Y)=A_{2} .
$$

Also the third moment of the random variable $Y$ is

$$
\rho_{3}=\sum_{m<u_{j} \leq n} u_{j}^{3} /\left(1+\mathrm{e}^{\sigma u_{j}}\right)
$$


Using the formula for the $n$th Fourier coefficient we have

$$
\mathbb{P}(Y=n)=\int_{-1 / 2}^{1 / 2} \varphi(\alpha) \mathrm{e}(-\alpha n) d \alpha .
$$

If an asymptotic result along the lines of the local limit theorem presented by Gnedenko and Kolmogorov [8, Section 49, page 233] held in the present situation we might hope that as $n \rightarrow \infty$,

$$
\mathbb{P}(Y=n)=\frac{1}{\sqrt{2 \pi \mathbb{V}(Y)}} \mathrm{e}^{-(n-\mathbb{E}(Y))^{2} / 2 \mathbb{V}(Y)}+o(1 / \sqrt{\mathbb{V}(Y)}) .
$$

If further we had $\mathbb{E}(Y)=n$, then, using (2.9) we would have

$$
\mathbb{P}(Y=n)=\frac{1}{\sqrt{2 \pi A_{2}}}+o\left(1 / \sqrt{A_{2}}\right),
$$

and since $A_{2} \rightarrow \infty$ as $n \rightarrow \infty$ (as will be evident from some lemmas estimating $A_{2}$ later in this paper), this implies

$$
\mathbb{P}(Y=n) \sim \frac{1}{\sqrt{2 \pi A_{2}}} \text { as } n \rightarrow \infty .
$$

By (2.6) and (2.9) we can ensure that $\mathbb{E}(Y)=n$ by choosing $\sigma$ so that

$$
n=\sum_{m<u_{j} \leq n} \frac{u_{j}}{1+\mathrm{e}^{\sigma u_{j}}}=A_{1} .
$$

We show later on that such a choice of $\sigma$ is possible.

This leads to the conjecture that, under suitable conditions on the sequence $u$ and on $m$, the result (2.11) will hold and hence, by (2.1), as $n \rightarrow \infty$,

$$
q_{u}(m, n) \sim \mathrm{e}^{\sigma n} \prod_{m<u_{j} \leq n}\left(1+\mathrm{e}^{-\sigma u_{j}}\right) \frac{1}{\sqrt{2 \pi A_{2}}},
$$

with $\sigma$ as in (2.12). This is the basis of the main theorem (Theorem 2 below) which under appropriate conditions will give an asymptotic estimate of $q_{u}(m, n)$ with the right hand side of (2.13) as its main term.

\section{Discussion of hypotheses}

For a given strictly increasing sequence of positive integers $u$, let the counting function $U$ with domain $[0, \infty)$ be defined by $U(x)=\operatorname{card}\left\{u_{j}: u_{j} \leq x\right\}$. We can now introduce the two hypotheses $H$ and $K$ on the sequence $u$ which turned out to be appropriate for the purposes of the main theorem. The first of these is as follows. 
HYPOTHESIS H. There are real constants $s, t, C_{0}$ satisfying $0<s \leq 1, t \geq 0$, $C_{0}>0$ such that,

$$
U(x) \sim C_{0} x^{s}(\log x)^{-t} \text { as } x \rightarrow \infty .
$$

It can be shown that Hypothesis $\mathrm{H}$ is equivalent to

$$
u_{j} \sim\left(s C_{0}\right)^{-1 / s} j^{1 / s}(\log j)^{t / s} \text { as } j \rightarrow \infty .
$$

It is clear that any sequence satisfying Hypothesis $\mathrm{H}$ must satisfy (RS1) given by Roth and Szekeres. We note also that Hypothesis $\mathrm{H}$ implies the existence of a positive constant $C_{1}$ depending only on $u$ such that

$$
U(x) \leq C_{1} x^{s}(\log x)^{-t} \text { for all } x \geq 2 .
$$

We note that by the Prime Number Theorem, the sequence of primes $u=\left(p_{j}\right)$ satisfies Hypothesis $\mathrm{H}$ with $s=t=1$. Also the sequence of positive $k$ th powers satisfies (3.2) and hence satisfies Hypothesis $\mathrm{H}$ with $s=1 / k, t=0$.

The second hypothesis, Hypothesis $\mathrm{K}$, is a further condition on sequences which already satisfy $\mathrm{H}$ and will be used only in estimating the supplementary integral $S$ in (4.4).

HYPOTHESIS K. Let $s$ be as in Hypothesis H. For every real number $\lambda \in(1,2)$, there are positive constants $x_{0}$ and $K_{0}$ (which depend only on $\lambda$ and the sequence $u$ ) such that for every $x>x_{0}$,

$$
\sum_{x<u_{j} \leq \lambda x}\left\|\alpha u_{j}\right\|^{2}>K_{0} x^{s(2-\lambda)}
$$

whenever $|\alpha| \in(1 /(2 \lambda x), 1 / 2)$. (Here \|\| denotes the distance from the nearest integer.)

Because of the inequality $2\|x\| \leq \sin \pi x \leq \pi\|x\|$ we observe that Hypothesis $\mathrm{K}$ is equivalent to the condition that for every $\lambda \in(1,2)$ and for every $x>x_{0}$,

$$
\sum_{x<u_{j} \leq \lambda x} \sin ^{2} \pi \alpha u_{j}>K_{0}^{\prime} x^{s(2-\lambda)}
$$

whenever $|\alpha| \in(1 /(2 \lambda x), 1 / 2)$, for some positive constants $x_{0}, K_{0}^{\prime}$ depending only on $\lambda$ and $u$. It is easily shown that any sequence satisfying Hypothesis $\mathrm{K}$ must satsify (RS2) of Roth and Szekeres.

In my thesis [5], I showed that sequences which satisfy both of the two Hypotheses $\mathrm{H}$ and $\mathrm{K}$ include the sequence $\left(j^{k}\right)$ of $k$ th powers for $k \geq 1$, the sequence $\left(p_{j}\right)$ of the primes, and, more generally, sequences of the form $(P(j))$ and $\left(P\left(p_{j}\right)\right)$ for fairly general polynomials $P(x)$, as well as certain uniformly distributed sequences. In the paper [4], I give full details of the proof that the sequence $\left(j^{k}\right)$ satisfies both $H$ and $K$. 


\section{The main theorem}

Convention on constants For given real valued functions $f$ and $g$ on $\mathbb{N}$ such that $g(n)>0$ for large integral $n$, we shall use $f(n)=O(g(n)), f(n) \ll g(n)$, $f(n) \sim g(n)$, and $f(n)=o(g(n))$ as $n \rightarrow \infty$, in their usual sense. We shall also sometimes write $f(n) \gg g(n)$ if $g(n) \ll f(n)$ and $f(n) \asymp g(n)$ if both $f(n) \ll g(n)$ and $f(n) \gg g(n)$ hold, as $n \rightarrow \infty$. Corresponding notation will apply for $f(x), g(x)$ for real $x$.

For the purposes of the main theorem, it will be important to identify all parameters on which the constants implied by the above notations may depend. These constants will normally depend on the particular sequence $u$ (or on the associated values of $C_{0}$, $s, t$ in Hypothesis $\mathrm{H}$ and hence on $u$ ), and any other parameters on which they may depend will be shown by subscripts. Thus, for example,

$$
f(n)=O_{a}(g(n)) \quad \text { as } n \rightarrow \infty, \quad \text { and } \quad f(n) \underset{a}{\ll} g(n) \quad \text { as } n \rightarrow \infty \text {, }
$$

both mean that there exist positive constants $C=C_{a}, n_{0}=n_{0}(a)$ which depend only on the sequence $u$ and the parameter $a$ such that $|f(n)| \leq C|g(n)|$ for every $n>n_{0}$. (However the status of the implied constants will be explicitly mentioned in the formal statements of results.)

Statement of main theorem We are now in a position to state the main theorem of this paper.

THEOREM 2. Let $u$ be a strictly increasing sequence of positive integers satisfying Hypotheses $\mathrm{H}$ and $\mathrm{K}$. Let $\delta$ be a positive constant such that

$$
0<\delta<1
$$

Let $q_{u}(m, n)$ be as in Section 1 and let $\sigma$ and $A_{2}$ be as in be as in (2.12) and (2.7). Then as $n \rightarrow \infty$,

$$
\begin{aligned}
q_{u}(m, n)= & \mathrm{e}^{\sigma n} \prod_{m<u j \leq n}\left(1+\mathrm{e}^{-\sigma u}\right) \frac{1}{\sqrt{2 \pi A_{2}}} \\
& \times\left(1+O\left(n^{-s /(2 s+2)}(\log n)^{-t /(2 s+2)}\right)+O_{\delta}\left((m / n)^{16 / 33}\right)\right),
\end{aligned}
$$

for $0 \leq m \leq n^{1-\delta}$, where the implied constants for the O-term depend only on $u$ and those for the $O_{\delta}$-term depend only on $u$ and $\delta$.

Outline of proof of main theorem The proof of Theorem 2 will be based on Lemma 1. Using the notation $\mathrm{e}(x)=\mathrm{e}^{2 \pi i x}$ for real $x$, we see from (2.1) that we must 
estimate the integral

$$
\int_{-1 / 2}^{1 / 2} \varphi(\alpha) \mathrm{e}(-\alpha n) d \alpha
$$

We write

$$
\int_{-1 / 2}^{1 / 2} \varphi(\alpha) \mathrm{e}(-\alpha n) d \alpha=M+S
$$

where

$$
M=\int_{-\alpha_{0}}^{\alpha_{0}} \varphi(\alpha) \mathrm{e}(-\alpha n) d \alpha
$$

is the main integral and

$$
S=\int_{\left\{\alpha: \alpha_{0} \leq|\alpha| \leq 1 / 2\right\}} \varphi(\alpha) \mathrm{e}(-\alpha n) d \alpha
$$

is the supplementary integral, and where $\alpha_{0}$ is a number in the interval $(0,1 / 2)$ which is to be chosen appropriately.

After commencing with some preliminary lemmas in Section 5 I give in Section 6 some lemmas on the size of the characteristic function $\varphi$ and in Section 7 use these to estimate the main integral $M$ :

$$
M=\frac{1}{\sqrt{2 \pi A_{2}}}\left(1+E_{1}\left(\alpha_{0}\right)\right)
$$

with an explicit upper bound for the error $E_{1}$. In Section $8 I$ give estimates of the variance $A_{2}$ and related quantities and in Section 9 give an upper bound for the supplementary integral $S$. The climax of this paper will be reached in Section 10 where the proof of Theorem 2 will be completed. Finally, in Section 11, a modification of the main theorem restricted to 'small' $m$ will be presented.

\section{Preliminary lemmas}

We commence with the following lemma which gives a consequence of Hypothesis $\mathrm{H}$, which will be used in the proof of subsequent lemmas.

LEMMA 3. Let $u$ be a strictly increasing sequence of positive integers satisfying Hypothesis $H$. Then there is a positive integer $n_{0}=n_{0}(u)$ depending only on u such that for $r>n_{0}$,

$$
2\left(1-2^{-s}\right) C_{0} r^{s}(\log r)^{-t} \geq U(r)-U(r / 2) \geq(1 / 2)\left(1-2^{-s}\right) C_{0} r^{s}(\log r)^{-t}
$$


ProOF. By Hypothesis $\mathrm{H}$, as $r \rightarrow \infty$,

$$
U(r) \sim C_{0} r^{s}(\log r)^{-t}, \quad U(r / 2) \sim 2^{-s} C_{0} r^{s}(\log r)^{-t} .
$$

So we have $U(r)-U(r / 2) \sim\left(1-2^{-s}\right) C_{0} r^{s}(\log r)^{-t}$ and the result follows easily.

The following lemma guarantees the existence of the parameter $\sigma$ defined in (2.12).

LEMMA 4. Let $u$ be a strictly increasing sequence of positive integers satisfying Hypothesis $H$. For integers $m$ and $n$ such that $0 \leq m<n / 2$, let

$$
A_{1}(x)=\sum_{m<u_{j} \leq n} \frac{u_{j}}{1+\mathrm{e}^{x u_{j}}}
$$

Then there is a positive number $n_{0}$ depending only on the sequence $u$ such that for every number $n>n_{0}$ a unique real number $\sigma=\sigma(m, n, u)$ exists such that $0<\sigma<1 / 4$ and $A_{1}(\sigma)=n$.

ProOF. Let $A_{1}(x)=\sum_{m<u_{j} \leq n} u_{j} /\left(1+\mathrm{e}^{x u_{j}}\right)$. Then $A_{1}$ is continuous and strictly decreasing on $\mathbb{R}$. Note that applying some elementary inequalities, Lemma 3 and Hypothesis $\mathrm{H}$ gives

$$
A_{1}(0)=\frac{1}{2} \sum_{m<u_{j} \leq n} u_{j} \geq \frac{1}{2} \sum_{n / 2<u_{j} \leq n} u_{j} \geq \frac{n}{4} \sum_{n / 2<u_{j} \leq n} 1 \geq \frac{C_{0}}{8} n^{1+s}(\log n)^{-t}\left(1-2^{-s}\right) .
$$

Also

$$
A_{1}(1 / 4) \leq \sum_{m<u, \leq n} u_{j} \mathrm{e}^{-u_{j} / 4} \leq \sum_{k=1}^{\infty} k \mathrm{e}^{-k / 4}=\mathrm{e}^{-1 / 4} /\left(1-\mathrm{e}^{-1 / 4}\right)^{2} .
$$

It follows that there exists $n_{0}$, depending only on $C_{0}, s$ and $t$ and hence only on $u$, such that for $n>n_{0}$ we have $A_{1}(0)>n>A_{1}(1 / 4)$. By the Intermediate Value Theorem, there is a unique $\sigma$ in the interval $(0,1 / 4)$ such that $A_{1}(\sigma)=n$.

Thus we confirm our definition of $\sigma$ as the unique real number which satisfies (2.12). We now state a lemma which gives bounds on $\sigma$.

LEMMA 5. Let $u$ be a strictly increasing sequence of positive integers satisfying Hypothesis $H$, with the constants $C_{0}, s$ and $t$ as in Hypothesis $H$. Let $m$ and $n$ be integers such that $0 \leq m<n / 2$ and let $\sigma$ be the unique number given by (2.12). Then there is a positive number $n_{0}$ depending only on the sequence $u$ such that for $n>n_{0}$ the following statements hold:

(i) We have $0<\sigma \ll n^{-1 /(1+s)}$. 
(ii) There is a positive constant $K_{1}=K_{1}\left(s, t, C_{0}\right)$ depending only on $s, t$ and $C_{0}$ such that

$$
\sigma>\frac{1}{2 r} \log \left(\frac{r^{1+s}(\log r)^{-t}}{n} \frac{\left(2^{s}-1\right) C_{0}}{4}\right)
$$

for $r \in \mathbb{Z}$ satisfying $\max \left(m, K_{1} n^{1 /(1+s)}(\log n)^{r /(1+s)}\right) \leq r \leq n / 2$.

PROOF. (i) We have that $\sigma>0$ from Lemma 4. We now prove the second inequality of (i). We have

$$
n=\sum_{m<u_{j} \leq n} \frac{u_{j}}{1+\mathrm{e}^{\sigma u_{j}}}<\sum_{j=1}^{\infty} u_{j} \mathrm{e}^{-\sigma u_{j}}
$$

where the series converges since $\sigma>0$. We note that by (3.3), since $t \geq 0$, we have $U(x) \leq C_{1} x^{s}$ for all $x \geq 2$. We use partial summation as in [11, Theorem $\mathrm{A}$, page 18] on the sum on the right above. Noting that for $f(x)=x \mathrm{e}^{-\sigma x}$ we have $-f^{\prime}(x)<\sigma x \mathrm{e}^{-\sigma x}$ and also that by (3.1) and also that by (3.1) $U(x) f(x)=o(1)$, we obtain

$$
\begin{aligned}
n<\sum_{j=1}^{\infty} u_{j} \mathrm{e}^{-\sigma u_{j}} & =-\int_{2}^{\infty} U(x) f^{\prime}(x) d x+\lim _{x \rightarrow \infty} f(x) U(x) \\
& <\int_{2}^{\infty} U(x) \sigma x \mathrm{e}^{-\sigma x} d x \ll \int_{2}^{\infty} \sigma x^{s+1} \mathrm{e}^{-\sigma x} d x .
\end{aligned}
$$

Using the change of variables $\sigma x=y$ on the last integral we see that

$$
n \ll\left(\frac{1}{\sigma}\right)^{s+1} \int_{2 / s}^{\infty} y^{s+1} \mathrm{e}^{-y} d y \leq\left(\frac{1}{\sigma}\right)^{s+1} \Gamma(s+2) \ll\left(\frac{1}{\sigma}\right)^{s+1},
$$

and hence $\sigma \ll n^{-1 /(1+s)}$.

(ii) Let $r \in \mathbb{Z}$ and $m \leq r \leq n / 2$. Using (2.12) together with the facts that $\sigma>0$ (by Lemma 4) and hence $\mathrm{e}^{\sigma x}>1$ for $x>0$, we obtain

$$
n \geq \sum_{r \leq u_{j}<2 r} \frac{u_{j}}{1+\mathrm{e}^{\sigma u_{j}}} \geq(U(2 r)-U(r)) \mathrm{e}^{-2 \sigma r}
$$

Using the lower bound for $U(2 r)-U(r)$ obtained from Lemma 3, taking logarithms and rearranging, we see that there is an $n_{0}=n_{0}(u)$ such that if $r>n_{0}$ then

$$
\sigma>\frac{1}{2 r} \log \left(\left(2^{x}-1\right) \frac{C_{0}}{4} \frac{r^{1+x}(\log r)^{-1}}{n}\right) .
$$


This inequality for $\sigma$ will improve upon $\sigma>0$ provided that

$$
\left(2^{s}-1\right) \frac{C_{0}}{4} \frac{r^{1+s}(\log r)^{-t}}{n}>1 .
$$

Using the fact that $r^{1+s}(\log r)^{-t}$ is strictly increasing for large enough $r$, it can be shown that (5.1) holds provided that $r>K_{1} n^{1 /(1+s)}(\log n)^{t /(1+s)}$, where $K_{1}=K_{1}(u)$ is a positive constant depending only on the sequence $u$.

As an immediate consequence we obtain the following lemma.

LEMMA 6. Let $u$ be a strictly increasing sequence of positive integers satisfying Hypothesis $H$. Let $m$ and $n$ be integers such that $0 \leq m<n / 2$. Let $\sigma$ be the unique number given by (2.12). Then as $n \rightarrow \infty$, on $\rightarrow \infty$ and $1 / \sigma \rightarrow \infty$, where the implied constants may depend on $s, t, C_{0}$ (and hence $u$ ) but not on $m$.

PROOF. Taking the reciprocal of the expressions in the inequality of Lemma 5 (i) gives $1 / \sigma \rightarrow \infty$. In part (ii) Lemma 5, taking $r=\lfloor n / 2\rfloor$ gives $\sigma n \rightarrow \infty$.

\section{Estimation of characteristic function}

The purpose of this section is to estimate the behaviour of the characteristic function defined in (2.2). We proceed to estimate $\varphi(\alpha)$ by examining its logarithm.

LEMMA 7. Let $u$ be a strictly increasing sequence of positive integers. Let $m$ and $n$ be integers such that $0 \leq m<n / 2$ and let $\sigma$ and $A_{2}$ be as in (2.12) and (2.7). Let $\alpha$ be a real number in the interval $(-1 / 2,1 / 2)$ and let $\varphi(\alpha)$ be as in (2.2). Suppose that

$$
\text { either }|\alpha|<\sigma /(2 \pi \log 3) \text { or } \sigma m>\log 3 \text {. }
$$

Then we have $\varphi(\alpha)=\mathrm{e}(\alpha n) \mathrm{e}^{-2 \pi^{2} \alpha^{2} A_{2}+R(\alpha)}$, where

$$
|R(\alpha)| \leq \frac{5}{3}|2 \pi \alpha|^{3} \rho_{3}
$$

and $\rho_{3}$ is in (2.10).

PROOF. We know from (2.2) and (2.3) that

$$
\log \varphi(\alpha)=\sum_{m<u_{j} \leq n} \log \left(p_{1 j}+p_{2 j} \mathrm{e}\left(\alpha u_{j}\right)\right)
$$


where $p_{1 j}, p_{2 j}$ are as in (2.4). Let $F_{j}(x)=\log \left(p_{1 j}+p_{2 j} \mathrm{e}\left(x u_{j}\right)\right)$ so that (6.3) becomes $\log \varphi(\alpha)=\sum_{m<u_{j} \leq n} F_{j}(\alpha)$. Taylor's Theorem with the integral form of the remainder (Spivak [15, page 346]) gives

$$
F_{j}(\alpha)=F_{j}(0)+\alpha F_{j}^{\prime}(0)+\frac{\alpha^{2}}{2} F_{j}^{\prime \prime}(0)+\frac{\alpha^{3}}{6} \int_{0}^{1}(1-v)^{2} F_{j}^{\prime \prime \prime}(v \alpha) d v .
$$

Now $F_{j}(0)=\log \left(p_{1 j}+p_{2 j}\right)=\log 1=0$. Also it is easily checked that

$$
\begin{aligned}
& F_{j}^{\prime}(0)=2 \pi i u_{j} p_{2 j}=2 \pi i f_{1}\left(u_{j}\right), \\
& F_{j}^{\prime \prime}(0)=\left(2 \pi i u_{j}\right)^{2}\left(p_{2 j}-p_{2 j}^{2}\right)=(2 \pi i)^{2} f_{2}\left(u_{j}\right),
\end{aligned}
$$

and

$$
\begin{aligned}
F_{j}^{\prime \prime \prime}(x)= & \left(2 \pi i u_{j}\right)^{3}\left(\frac{1}{1+\mathrm{e}^{\sigma u_{j}} \mathrm{e}\left(-u_{j} x\right)}-\frac{3}{\left(1+\mathrm{e}^{\sigma u_{j}} \mathrm{e}\left(-u_{j} x\right)\right)^{2}}\right. \\
& \left.+\frac{2}{\left(1+\mathrm{e}^{\sigma u_{j}} \mathrm{e}\left(-u_{j} x\right)\right)^{3}}\right) .
\end{aligned}
$$

We shall estimate the integral in (6.4), namely $\int_{0}^{1}(1-v)^{2} F_{j}^{\prime \prime \prime}(v \alpha) d v$, by first estimating an integral of the form

$$
\int_{0}^{1} \frac{(1-v)^{2}}{\left(1+\mathrm{e}^{\sigma u_{j}} \mathrm{e}\left(-u_{j} v \alpha\right)\right)^{l}} d v
$$

where $l \in \mathbb{N}$ (we will be taking only $l=1,2,3$ ). We observe that

$$
1+\mathrm{e}^{\sigma u_{j}} \mathrm{e}\left(-u_{j} v \alpha\right)=\mathrm{e}^{\sigma u_{j}} \mathrm{e}\left(-u_{j} v \alpha\right)\left(1+\mathrm{e}^{-\sigma u_{j}}\right)\left(1+\frac{2 i \sin \left(\pi \alpha v u_{j}\right) \mathrm{e}\left(\alpha v u_{j} / 2\right)}{1+\mathrm{e}^{\sigma u_{j}}}\right) .
$$

Further, for $j$ such that $m<u_{j} \leq n$, we observe the following.

(a) If $\sigma u_{j}>\log 3$, then $2 /\left(1+\mathrm{e}^{\sigma u_{j}}\right)<1 / 2$.

(b) If $\sigma u_{j} \leq \log 3$, then $\sigma m \leq \log 3$ and hence by (6.1),

$$
\left|\sin \left(\pi \alpha v u_{j}\right)\right| \leq \pi|\alpha| u_{j} \leq \pi \frac{|\alpha|}{\sigma} \sigma u_{j}<\frac{1}{2} .
$$

By virtue of the two preceding observations,

and so

$$
\left|\frac{2 i \sin \left(\pi \alpha v u_{j}\right) \mathrm{e}\left(\alpha v u_{j} / 2\right)}{1+\mathrm{e}^{\sigma u_{j}}}\right|<\frac{1}{2}
$$

$$
\left|1+\mathrm{e}^{\sigma u_{j}} \mathrm{e}\left(-u_{j} v \alpha\right)\right|>\left(1+\mathrm{e}^{\sigma u_{j}}\right)\left(1-\left|\frac{2 i \sin \left(\pi \alpha v u_{j}\right) \mathrm{e}\left(\alpha v u_{j} / 2\right)}{1+\mathrm{e}^{\sigma u_{j}}}\right|\right)>\frac{1+\mathrm{e}^{\sigma u_{j}}}{2} .
$$


Consequently for a positive integer $l$,

$$
\left|\int_{0}^{1} \frac{(1-v)^{2}}{\left(1+\mathrm{e}^{\sigma u_{j}} \mathrm{e}\left(-u_{j} v \alpha\right)\right)^{l}} d v\right|<\int_{0}^{1} \frac{|1-v|^{2}}{\left(1+\mathrm{e}^{\sigma u_{j}}\right)^{l}} 2^{l} d v=\frac{1}{3} 2^{l}\left(1+\mathrm{e}^{\sigma u_{j}}\right)^{-l}
$$

and using (6.7) we deduce that

$$
\begin{aligned}
\frac{1}{6}\left|\int_{0}^{1}(1-v)^{2} F_{j}^{\prime \prime \prime}(v \alpha) d v\right| & \leq \frac{\left(2 \pi i u_{j}\right)^{3}}{6}\left(1+\mathrm{e}^{\sigma u_{j}}\right)^{-1}\left(\frac{2}{3}+3 \cdot \frac{4}{3}+2 \cdot \frac{8}{3}\right) \\
& =\frac{5}{3} \frac{\left(2 \pi u_{j}\right)^{3}}{1+\mathrm{e}^{\sigma u_{j}}} .
\end{aligned}
$$

It follows from (6.4)-(6.6) and (6.8) that

$$
F_{j}(\alpha)=2 \pi i \alpha f_{1}\left(u_{j}\right)+(2 \pi i \alpha)^{2} f_{2}\left(u_{j}\right) / 2+R_{j}(\alpha),
$$

where $\left|R_{j}(\alpha)\right| \leq(5 / 3)|2 \pi \alpha|^{3} u_{j}^{3} /\left(1+\mathrm{e}^{\sigma u_{j}}\right)$ and where $f_{1}(x)$ and $f_{2}(x)$ are defined as in (2.8). Summing both sides of (6.9) over $u_{j} \in(m, n]$ and using (2.6), (2.7) and (2.12) gives the result.

LEMMA 8. Let $u$ be a strictly increasing sequence of positive integers. Let $m$ and $n$ be integers such that $0 \leq m<n / 2$ and let $\sigma$ be as in (2.12). Let $\varphi(\alpha)$ be the characteristic function as in (2.2). There is a positive number $n_{0}$ depending only on $u$ such that for $n>n_{0}$ and for all real numbers $\alpha$,

$$
|\varphi(\alpha)| \leq \exp \left(-2 \sum_{m<u_{j} \leq n} \mathrm{e}^{-\sigma u_{j}}\left\|\alpha u_{j}\right\|^{2}\right) .
$$

ProOF. It is easily shown (see Freiman and Pitman [7, Lemma 3 (i)]) that

$$
\left|\varphi_{j}(\alpha)\right|^{2}=1-4 p_{1 j} p_{2 j} \sin ^{2}\left(\pi \alpha u_{j}\right) \leq \exp \left(-4 p_{1 j} p_{2 j} \sin ^{2}\left(\pi \alpha u_{j}\right)\right) .
$$

Now from (2.4) and positivity of $\sigma$ by Lemma 4, we have

$$
p_{1 j} p_{2 j}=\frac{\mathrm{e}^{-\sigma u_{j}}}{\left(1+\mathrm{e}^{-\sigma u_{j}}\right)^{2}}>\frac{1}{4} \mathrm{e}^{-\sigma u_{j}} .
$$

Using (2.2) and $|\sin \pi x| \geq 2\|x\|$ we obtain

$$
|\varphi(\alpha)|^{2} \leq \prod_{m<u_{j} \leq n} \exp \left(-4 \mathrm{e}^{-\sigma u_{j}}\left\|\alpha u_{j}\right\|^{2}\right)
$$

and the result follows. 


\section{Estimation of the main integral}

Let $\alpha_{0}$ be a number in the interval $(0,1 / 2)$ which will be chosen appropriately later on. Lemma 7 suggests that the integrand of the main integral $M$, as in (4.3), is approximated by the probability density function of a normal random variable with variance $A_{2}$. By using a suitable choice of $\alpha_{0}$ together with Lemma 8 and Hypothesis $\mathrm{K}$ it will be possible to show that the supplementary integral is dominated by the main integral. We show that the main integral $M$ is approximated fairly accurately by the integral $\int_{-\infty}^{\infty} \mathrm{e}^{-2 \pi^{2} A_{2} \alpha^{2}} d \alpha$.

LEMMA 9. For a given strictly increasing sequence $u$ of positive integers and for given integers $m$ and $n$ such that $0 \leq m<n / 2$, let $\sigma, A_{2}, \rho_{3}$ be as in (2.12), (2.7), (2.10). Suppose that a given real number $\alpha_{0}$ in the interval $(0,1 / 2)$ satisfies the conditions

$$
\text { either } \begin{gathered}
\alpha_{0}<\sigma /(2 \pi \log 3) \quad \text { or } \sigma m>\log 3, \\
(5 / 3)\left(2 \pi \alpha_{0}\right)^{3} \rho_{3}<1 / 2 \text { and } \\
\alpha_{0}>1 /\left(2 \pi \sqrt{A_{2}}\right) .
\end{gathered}
$$

Then there is a positive number $n_{0}$ depending only on $u$ such that for $n>n_{0}$ the main integral $M=\int_{-\alpha_{0}}^{\alpha_{0}} \varphi(\alpha) \mathrm{e}(-\alpha n) d \alpha$ satisfies

$$
M=\frac{1}{\sqrt{2 \pi A_{2}}}\left(1+E_{1}\left(\alpha_{0}\right)\right)
$$

where $E_{1}\left(\alpha_{0}\right) \ll\left(\rho_{3} / A_{2}^{3 / 2}\right)+\mathrm{e}^{-2 \pi^{2} A_{2} \alpha_{0}^{2}}$ and the implied constants depend only on $u$.

Proof. We first show

$$
M=\int_{-\alpha_{0}}^{\alpha_{0}} \mathrm{e}^{-2 \pi^{2} A_{2} \alpha^{2}} d \alpha+O\left(\frac{\rho_{3}}{A_{2}^{2}}\right)
$$

where the implied constants depend only on $u$. Substituting the expression for the characteristic function $\varphi(\alpha)$ given in Lemma 7 into the expression for the main integral $M$ in (4.3) gives

$$
M=\int_{-\alpha_{0}}^{\alpha_{0}} \exp \left(-2 \pi^{2} \alpha^{2} A_{2}+R(\alpha)\right) d \alpha
$$

Combining (6.2) with our assumption (7.2) ensures that $|R(\alpha)|<1 / 2$ and hence

$$
|\exp (R(\alpha))-1| \leq \sum_{l=1}^{\infty} \frac{|R(\alpha)|^{l}}{l !} \leq \frac{|R(\alpha)|}{1-|R(\alpha)|}<2|R(\alpha)| .
$$


Using (6.2) again gives $|\exp (R(\alpha))-1| \leq 2(5 / 3)|2 \pi \alpha|^{3} \rho_{3}$. Hence

$$
\begin{aligned}
\left|M-\int_{-\alpha_{0}}^{\alpha_{0}} \mathrm{e}^{-2 \pi^{2} A_{2}} d \alpha\right| & =\left|\int_{-\alpha_{0}}^{\alpha_{0}} \mathrm{e}^{-2 \pi^{2} \alpha^{2} A_{2}}(\exp (R(\alpha))-1) d \alpha\right| \\
& \leq \int_{-\alpha_{0}}^{\alpha_{0}} \mathrm{e}^{-2 \pi^{2} \alpha^{2} A_{2}} 2 \cdot \frac{5}{3}|2 \pi \alpha|^{3} \rho_{3} d \alpha .
\end{aligned}
$$

Using the change of variable $\beta=2 \pi \sqrt{A_{2}} \alpha$, writing $\beta_{0}=2 \pi \sqrt{A_{2}} \alpha_{0}$, and noting that $\beta_{0}>1$ by (7.3), we see that the integral on the right is

$$
\frac{\rho_{3}}{A_{2}^{3 / 2}} \frac{1}{2 \pi \sqrt{A_{2}}} \int_{-\beta_{0}}^{\beta_{0}} \exp \left(-\beta^{2} / 2\right)|\beta|^{3} d \beta \ll \frac{\rho_{3}}{A_{2}^{2}} .
$$

This completes the proof of (7.4).

We now show that

$$
\int_{-\alpha_{0}}^{\alpha_{0}} \mathrm{e}^{-2 \pi^{2} A_{2} \alpha^{2}} d \alpha=\int_{-\infty}^{\infty} \mathrm{e}^{-2 \pi^{2} A_{2} \alpha^{2}} d \alpha+O\left(\frac{1}{\sqrt{A_{2}}} \exp \left(-2 \pi^{2} \alpha_{0}^{2} A_{2}\right)\right)
$$

where the implied constants depend only on $u$.

We have

$$
\int_{-\alpha_{0}}^{\alpha_{0}} \mathrm{e}^{-2 \pi^{2} A_{2} \alpha^{2}} d \alpha=\int_{-\infty}^{\infty} \mathrm{e}^{-2 \pi^{2} A_{2} \alpha^{2}} d \alpha+2 \int_{\alpha_{0}}^{\infty} \exp \left(-2 \pi^{2} \alpha^{2} A_{2}\right) d \alpha
$$

Writing $\beta_{0}=2 \pi \sqrt{A_{2}} \alpha_{0}$, noting that $\beta_{0}>1$ by (7.3) and using the change of variable $\beta=2 \pi \sqrt{A_{2}} \alpha$, we see that the integral on the right is

$$
\frac{1}{\pi \sqrt{A_{2}}} \int_{\beta_{0}}^{\infty} \mathrm{e}^{-\beta^{2} / 2} d \beta<\frac{1}{\pi \sqrt{A_{2}}} \int_{\beta_{0}}^{\infty} \beta \mathrm{e}^{-\beta^{2} / 2} d \beta=\frac{1}{\pi \sqrt{A_{2}}} \exp \left(-2 \pi^{2} A_{2} \alpha_{0}^{2}\right),
$$

and this proves (7.5).

From (7.4) and (7.5) we have

$$
M=\int_{-\infty}^{\infty} \mathrm{e}^{-2 \pi^{2} A_{2} \alpha^{2}} d \alpha+O\left(\frac{\rho_{3}}{A_{2}^{2}}\right) O\left(\frac{1}{\sqrt{A_{2}}} \exp \left(-2 \pi^{2} \alpha_{0}^{2} A_{2}\right)\right)
$$

which gives the required result.

It will turn out that there is a choice of $\alpha_{0}$ which is compatible with the conditions (7.1)-(7.3). 


\section{Estimation of cumulants}

In view of the above lemma, we require an estimate of the second cumulant $A_{2}$ and the quantity $\rho_{3}$. In this section we estimate the cumulants $A_{1}, A_{2}$ and the related quantities $\sigma$ and $\rho_{3}$. We start with some general estimates of integrals and sums.

LEMMA 10. Let $m$ and $n$ be integers such that $0 \leq m<n / 2$ and let $\sigma$ be as in (2.12). Let $a, b$ be positive real numbers and let $r \in \mathbb{N}$ such that $(1 / \sigma) \max (1,2 a) \leq r \leq n$. Then

$$
\int_{r}^{\infty} x^{a}(\log x)^{-b} \mathrm{e}^{-\sigma x} d x \underset{a}{\ll} \frac{1}{\sigma}\left(\log \frac{1}{\sigma}\right)^{-b} r^{a} \mathrm{e}^{-\sigma r}
$$

where the implied constants depend only on the real number $a$.

ProOF. By using the change of variables $y=\sigma x$ we obtain

$$
\begin{aligned}
\int_{r}^{\infty} x^{a}(\log x)^{-b} \mathrm{e}^{-\sigma x} d x & =\sigma^{-a-1} \int_{\sigma r}^{\infty} y^{a}\left(\log \frac{y}{\sigma}\right)^{-b} \mathrm{e}^{-y} d y \\
& =\sigma^{-a-1} \int_{\sigma r}^{\infty} y^{a} \frac{1}{\left(\log y+\log \frac{1}{\sigma}\right)^{b}} \mathrm{e}^{-y} d y .
\end{aligned}
$$

Since $\sigma r \geq 1$, the right-hand side is

$$
\begin{aligned}
& \leq \sigma^{-a-1} \int_{\sigma r}^{\infty} y^{a}\left(\log \frac{1}{\sigma}\right)^{-b} \mathrm{e}^{-y} d y \\
& =\sigma^{-a-1}\left(\log \frac{1}{\sigma}\right)^{-b} \int_{\sigma r}^{\infty} y^{a} \mathrm{e}^{-y} d y .
\end{aligned}
$$

The result now follows since for $\sigma r \geq 2 a$ the integral on the right is at most $K(\sigma r)^{a} \mathrm{e}^{-\sigma r}$.

LEMMA 11. Let $a, b, z$ be positive real numbers such that $z>1$. Then

$$
J=J(z ; a, b)=\int_{2}^{\infty} x^{a}(\log x)^{-b} \mathrm{e}^{-x / z} d x \underset{a, b}{\ll} z^{a+1}(\log z)^{-b} .
$$

Proof. Using the change of variables $y=x / z$ and the fact that $z$ is positive we have

$$
J=z^{a+1} \int_{2 / z}^{\infty} y^{a}(\log y z)^{-b} \mathrm{e}^{-y} d y .
$$


Case 1: Suppose $z>2$. We write

$$
J=z^{a+1}\left(\int_{2 / z}^{1}+\int_{1}^{\infty}\right) y^{a}(\log y z)^{-b} \mathrm{e}^{-y} d y .
$$

Now since $\log :>0$ for $y \in(1, \infty)$ and since $\log z>0$,

$$
\begin{aligned}
\int_{1}^{\infty} y^{a}(\log y+\log z)^{-b} \mathrm{e}^{-y} d y & <(\log z)^{-b} \int_{0}^{\infty} y^{a} \mathrm{e}^{-y} d y \\
& =\Gamma(a+1)(\log z)^{-b}
\end{aligned}
$$

The integral over the interval $(2 / z, 1)$ is

$$
\int_{2 / z}^{1} y^{a}(\log y z)^{-b} \mathrm{e}^{-y} d y=(\log z)^{-b} \int_{2 / z}^{1} y^{a}\left(1-\frac{\log (1 / y)}{\log z}\right)^{-b} \mathrm{e}^{-y} d y .
$$

In order to estimate the integrand on the right, we use the easily checked result that for positive $c, r, w$, the inequality $w<c-r$ implies that

$$
\left(1-\frac{w}{c}\right)^{-1}<1+\frac{w}{r}
$$

For $2 / z<y<1$, we have $0<\log (1 / y)<\log z-\log 2$ and so by the above inequality

$$
\left(1-\frac{\log (1 / y)}{\log z}\right)^{-1}<1+\frac{\log (1 / y)}{\log 2} \text {. }
$$

Using this bound on the right hand side of (8.3) we obtain

$$
\int_{2 / z}^{1} y^{a}(\log y z)^{-b} \mathrm{e}^{-y} d y<(\log z)^{-b} \int_{0}^{1} y^{a}\left(1-\frac{\log (1 / y)}{\log z}\right)^{-b} \mathrm{e}^{-y} d y \underset{a, b}{\ll}(\log z)^{-b}
$$

since the integrand on the right is bounded as $y \rightarrow 0$.

By (8.1), this inequality together with (8.2) gives the result.

Case 2: Suppose $1<z \leq 2$. Then $J<z^{a+1} \int_{1}^{\infty} y^{a}(\log y z)^{-b} \mathrm{e}^{-y} d y$ and the result follows from (8.2).

The following lemma will enable us to give upper bounds for $\rho_{1}, \rho_{2}$, and $\rho_{3}$ for all $m$ in the range $0 \leq m<n / 2$ and to find bounds on these quantities for large $m$, that is for $m \geq 1 / \sigma$.

LEMMA 12. Let $u$ be a strictly increasing sequence of positive integers satisfying Hypothesis $H$ with $C_{n}$, s and $t$ being as in Hypothesis $H$. Let $m$ and $n$ be integers such that $0 \leq m<n / 2$ and let $\sigma$ be as in (2.12). Let $k$ be a fixed positive integer and $\lambda a$ fuxed real number such that $1<\lambda<2$. Then the following estimates hold, with the implied constants depending only on $u$ and the parameters shown. 
(i) As $n \rightarrow \infty$,

$$
\sum_{0<u_{j} \leq n} u_{j}^{k} \mathrm{e}^{-\sigma u_{j}} \ll_{k}\left(\frac{1}{\sigma}\right)^{s+k}\left(\log \frac{1}{\sigma}\right)^{-t} .
$$

(ii) Let $r$ be a positive integer such that $2(k+s) / \sigma \leq r \leq n / \lambda$. Then as $n \rightarrow \infty$,

$$
r^{k+s}(\log r)^{-t} \mathrm{e}^{-\sigma r \lambda} \underset{k, \lambda}{\ll} \sum_{r<u_{j} \leq n} u_{j}^{k} \mathrm{e}^{-\sigma u_{j}} \ll_{k} r^{k+s}(\log r)^{-t} \mathrm{e}^{-\sigma r} .
$$

PrOOF. (i) We have that

$$
\sum_{0<u_{j} \leq n} u_{j}^{k} \mathrm{e}^{-\sigma u_{j}}<O(1)+\sum_{2<u_{j}<\infty} u_{j}^{k} \mathrm{e}^{-\sigma u_{j}} .
$$

Let $\phi(x)=x^{k} \mathrm{e}^{-\sigma x}$, hence $\phi^{\prime}(x)=\left(k x^{k-1}-\sigma x^{k}\right) \mathrm{e}^{-\sigma x}$. Then, since $U(x) \phi(x) \rightarrow 0$ as $x \rightarrow \infty$, partial summation as in [11, Theorem A, page 18], gives

$$
\sum_{2<u_{j}<\infty} \phi\left(u_{j}\right)=-\int_{2}^{\infty} U(x) \phi^{\prime}(x) d x-U(2) \phi(2) .
$$

We immediately have the inequality

$$
\begin{aligned}
-\int_{2}^{\infty} & U(x) \phi^{\prime}(x) d x-U(2) \phi(2) \\
& =-\int_{2}^{\infty} U(x)\left(k x^{k-1}-\sigma x^{k}\right) \mathrm{e}^{-\sigma x} d x-U(2) \phi(2) \\
& <\int_{2}^{\infty} U(x) \sigma x^{k} \mathrm{e}^{-\sigma x} d x
\end{aligned}
$$

Since Hypothesis $\mathrm{H}$ holds, it follows from (3.3) and the comments preceding it that

$$
\int_{2}^{\infty} U(x) x^{k} \mathrm{e}^{-\sigma x} d x \ll \int_{2}^{\infty} x^{s+k}(\log x)^{-1} \mathrm{e}^{-\sigma x} d x
$$

By Lemma 11 (with $z=1 / \sigma$, this is, $>1$ by Lemma 4 )

and so

$$
\int_{2}^{\infty} x^{s+k}(\log x)^{-t} \mathrm{e}^{-\sigma x} d x \underset{s, k, t}{\ll}\left(\frac{1}{\sigma}\right)^{s+k+1}\left(\log \frac{1}{\sigma}\right)^{-t},
$$

$$
\int_{2}^{\infty} U(x) \sigma x^{k} \mathrm{e}^{-\sigma x} d x \underset{k}{\ll}\left(\frac{1}{\sigma}\right)^{s+k}\left(\log \frac{1}{\sigma}\right)^{-t}
$$


where the implied constant depends only on $k$ and the sequence $u$. The result follows immediately from (8.4)-(8.6).

(ii) By partial summation again,

$$
\sum_{r<u_{j}<\infty} u_{j}^{k} \mathrm{e}^{-\sigma u_{j}}=-\int_{r}^{\infty} U(x) \phi^{\prime}(x) d x-\phi(r) U(r),
$$

where $\phi(x)=x^{k} \mathrm{e}^{-\sigma x}$. Thus

$$
\begin{aligned}
\sum_{r<u_{j} \leq n} u_{j}^{k} \mathrm{e}^{-\sigma u_{j}} & \leq-\int_{r}^{\infty} U(x) k x^{k-1} \mathrm{e}^{-\sigma x} d x+\int_{r}^{\infty} U(x) \sigma x^{k} \mathrm{e}^{-\sigma x} d x-\phi(r) U(r) \\
& <\int_{r}^{\infty} U(x) \sigma x^{k} \mathrm{e}^{-\sigma x} d x .
\end{aligned}
$$

Now as $n \rightarrow \infty$, we have that $r \rightarrow \infty$ because $r \geq 2(k+s) / \sigma$ and Lemma 6 gives us that $1 / \sigma \rightarrow \infty$. Using Hypothesis $\mathrm{H}$ it is easily seen that as $n \rightarrow \infty$

$$
\int_{r}^{\infty} U(x) \sigma x^{k} \mathrm{e}^{-\sigma x} d x \sim \int_{r}^{\infty} C_{0} \sigma x^{k+s}(\log x)^{-t} \mathrm{e}^{-\sigma x} d x,
$$

which in view of Lemma 10 gives

$$
\int_{r}^{\infty} U(x) \sigma x^{k} \mathrm{e}^{-\sigma x} d x \ll\left(\log \frac{1}{\sigma}\right)^{-t} r^{k+s} \mathrm{e}^{-\sigma r} .
$$

Since $2(k+s) / \sigma \leq r$, this completes the proof of the second inequality of (ii).

We know that $\lambda r \leq n$ because $r \leq n / \lambda$ so that we may write

$$
\sum_{r<u_{j} \leq n} u_{j}^{k} \mathrm{e}^{-\sigma u_{j}} \geq \sum_{r<u_{j} \leq \lambda r} u_{j}^{k} \mathrm{e}^{-\sigma u_{j}}
$$

Now for $r<u_{j} \leq \lambda r$ we have that $u_{j}^{k}>r^{k}$ and $\mathrm{e}^{-\sigma u_{j}} \geq \mathrm{e}^{-\sigma r \lambda}$ and so the right-hand side above is

$$
>r^{k} \mathrm{e}^{-\sigma r \lambda} \sum_{r<u j \leq \lambda r} 1
$$

Also by Hypothesis $\mathrm{H}$ we have that as $r \rightarrow \infty$

$$
\sum_{r<u_{j} \leq \lambda r} 1=U(\lambda r)-U(r) \sim C_{0}\left(\lambda^{s}-1\right) r^{s}(\log r)^{-t}
$$

Thus we have that

$$
\sum_{r<u, \leq \lambda r} u_{j}^{k} \mathrm{e}^{-\sigma u,} \gg_{\lambda} \mathrm{e}^{-\sigma r \lambda} r^{k} r^{s}(\log r)^{-1}
$$

which gives the first inequality in (ii). 
The following lemma gives upper bounds for $A_{1}, A_{2}$ and $\rho_{3}$ in terms of $\sigma$ which are valid for all $m$, together with estimates of these quantities which are valid when $m$ is 'large'.

COROLLARY 13. Let $u$ be a strictly increasing sequence of positive integers satisfying Hypothesis $H$ and let $s$ and $t$ be as in Hypothesis $H$. Let $m$ and $n$ be integers such that $0 \leq m<n / 2$ and let $\sigma$ be as in (2.12). Let $A_{1}, A_{2}$ and $\rho_{3}$ be defined as in (2.6), (2.7), and (2.10). Then we have the following estimates.

(i) As $n \rightarrow \infty$,

$$
n=A_{1} \ll \sigma^{-1-s}\left(\log \frac{1}{\sigma}\right)^{-t}, \quad A_{2} \ll \sigma^{-2-s}\left(\log \frac{1}{\sigma}\right)^{-t}, \quad \rho_{3} \ll \sigma^{-3-s}\left(\log \frac{1}{\sigma}\right)^{-t},
$$

where the implied constants depend on $u$ only.

(ii) Let $\lambda$ be a given real number in the interval $(1,2)$. Then as $n \rightarrow \infty$,

$$
\begin{aligned}
& m^{1+s}(\log m)^{-t} \mathrm{e}^{-\sigma m \lambda} \underset{\lambda}{\ll} A_{1} \ll m^{1+s}(\log m)^{-t} \mathrm{e}^{-\sigma m}, \\
& m^{2+s}(\log m)^{-t} \mathrm{e}^{-\sigma m \lambda} \underset{\lambda}{\ll} A_{2} \ll m^{2+s}(\log m)^{-t} \mathrm{e}^{-\sigma m}, \\
& m^{3+s}(\log m)^{-t} \mathrm{e}^{-\sigma m \lambda} \underset{\lambda}{\ll} \rho_{3} \ll m^{3+s}(\log m)^{-t} \mathrm{e}^{-\sigma m},
\end{aligned}
$$

for $2(3+s) / \sigma \leq m \leq n / 2$.

ProOF. (i) Since

$$
A_{1}<\sum_{m<u_{j} \leq n} u_{j} \mathrm{e}^{-\sigma u_{j}}, \quad A_{2}<\sum_{m<u_{j} \leq n} u_{j}^{2} \mathrm{e}^{-\sigma u_{j}}, \quad \rho_{3}<\sum_{m<u_{j} \leq n} u_{j}^{3} \mathrm{e}^{-\sigma u_{j}}
$$

we immediately have the required inequalities upon application of Lemma 12 (i).

(ii) Combining Lemma 12 (ii) with the observations that as $n \rightarrow \infty$,

$$
n=A_{1} \asymp \sum_{m<u_{j} \leq n} u_{j} \mathrm{e}^{-\sigma u_{1}}, \quad A_{2} \asymp \sum_{m<u_{j} \leq n} u_{j}^{2} \mathrm{e}^{-\sigma u_{j}}, \quad \rho_{3} \asymp \sum_{m<u_{j} \leq n} u_{j}^{3} \mathrm{e}^{-\sigma u_{j}}
$$

for $1 / \sigma \ll m \leq n / 2$, where the implied constants depend only on $u$, gives the inequalities for $2(3+s) / \sigma \leq m \leq n / 2$.

The following lemma gives lower bounds for the quantities $A_{1}, A_{2}$ and $\rho_{3}$ in terms of the quantity $\sigma$ when $m$ is 'small'.

LEMMA 14. Let $u$ be a strictly increasing sequence of positive integers satisfying Hypothesis $H$ and let $s$ and $t$ be as in Hypothesis $H$. Let $m$ and $n$ be integers such 
that $0 \leq m<n / 2$ and let $\sigma$ be as in (2.12). Let $A_{1}, A_{2}$ and $\rho_{3}$ be defined as in (2.6), (2.7), and (2.10). Let $K \geq 1$ be a constant. Then as $n \rightarrow \infty$,

$$
\begin{gathered}
n=A_{1} \gg(1 / \sigma)^{s+1}(\log 1 / \sigma)^{-t}, \quad A_{2} \gg(1 / \sigma)^{s+2}(\log 1 / \sigma)^{-t}, \\
\rho_{3} \gg(1 / \sigma)^{s+3}(\log 1 / \sigma)^{-t},
\end{gathered}
$$

for $0 \leq m \leq K / \sigma$, where the implied constants depend only on $K$ and the sequence $u$.

ProOF. We prove the first inequality and note that the proof of the remaining two inequalities follow a similar line of reasoning. Using (2.6) and noting that $m \leq K / \sigma$ and $\left(1+\mathrm{e}^{\sigma u_{j}}\right)^{-1}>\mathrm{e}^{-\sigma u_{j}} / 2$ gives

$$
A_{1}=\sum_{m<u_{j} \leq n} \frac{u_{j}}{1+\mathrm{e}^{\sigma u_{j}}}>\frac{1}{2} \sum_{K / \sigma<u_{j} \leq n} u_{j} \mathrm{e}^{-\sigma u_{j}} .
$$

By Lemma 12 (ii) with $\lambda=3 / 2$ and $r=\lceil K / \sigma\rceil$, this is,

$$
\begin{aligned}
& \gg(K / \sigma)^{s+1}(\log K / \sigma)^{-t} \mathrm{e}^{-\sigma(K / \sigma) \cdot 3 / 2} \\
& \gg(1 / \sigma)^{s+1}(\log 1 / \sigma)^{-t},
\end{aligned}
$$

where the implied constant depends only on $K$ and the sequence $u$.

With the following lemma, we are well on the way to obtaining explicit estimates of the quantities $A_{2}$ and $\rho_{3}$.

LEMMA 15. Let $u$ be a strictly increasing sequence of positive integers satisfying Hypothesis $H$ and let $s$ and $t$ be as in Hypothesis $H$. Let $m$ and $n$ be integers such that $0 \leq m<n / 2$ and let $\sigma$ be as in (2.12). Let $A_{1}, A_{2}$ and $\rho_{3}$ be defined as in (2.6), (2.7), and (2.10). Let $\lambda$ be a real number in the interval $(1,2)$. Then as $n \rightarrow \infty$,

$$
m \ll \frac{A_{2}}{A_{1}} \ll \frac{\rho_{3}}{A_{2}} \underset{\lambda}{\ll} m \mathrm{e}^{\sigma m(\lambda-1)},
$$

for $(6+2 s) / \sigma \leq m \leq n / 2$, where the first and second implied constants are absolute and the third implied constant depends only on $\lambda$ and the sequence $u$.

PROOF. For a positive integer $k$, let $\rho_{k}=\sum_{m<u_{j} \leq n} u_{j}^{k} /\left(1+\mathrm{e}^{\sigma u_{\prime}}\right)$, so that $\rho_{3}$ is given by $(2.10)$ and from (2.6) and (2.7) we have

$$
\rho_{1}=A_{1}, \quad \rho_{2} / 2<A_{2}<\rho_{2} .
$$


We now show that

$$
m<\frac{\rho_{2}}{\rho_{1}} \leq \frac{\rho_{3}}{\rho_{2}} \underset{\lambda}{\ll} \mathrm{e}^{\sigma m(\lambda-1)},
$$

where the implied constant depends only on $\lambda$ and the sequence $u$. In view of (8.7) it is sufficient to show (8.8) in order to prove the lemma.

Firstly,

$$
m \rho_{1}=\sum_{m<u_{j} \leq n} \frac{m u_{j}}{1+\mathrm{e}^{\sigma u_{j}}}<\sum_{m<u_{j} \leq n} \frac{u_{j}^{2}}{1+\mathrm{e}^{\sigma u_{j}}}=\rho_{2} .
$$

Secondly, by applying the Cauchy-Schwarz inequality to the sum $\rho_{2}$ and writing $u_{j}^{2}=u_{j}^{1 / 2} u_{j}^{3 / 2}$ it is easily seen that

$$
\rho_{2}^{2} \leq \rho_{1} \rho_{3}
$$

Finally, to show the third inequality in (8.8) we appeal to Corollary 13 (ii) to give as $n \rightarrow \infty$,

$$
\frac{\rho_{3}}{\rho_{2}} \asymp \frac{\rho_{3}}{A_{2}} \underset{\lambda}{\ll} m \mathrm{e}^{\sigma m(\lambda-1)},
$$

for $(6+2 s) / \sigma \leq m$ and this completes the proof of the lemma.

The following lemma provides an estimate of the quantity $\mathrm{e}^{\sigma m}$ which in tandem with Lemma 15 is of use in the estimation of the cumulant $A_{2}$ and the quantity $\rho_{3}$.

LEMMA 16. Let $u$ be a strictly increasing sequence of positive integers satisfying Hypothesis $H$ and let $s$ and $t$ be as in Hypothesis $H$. Let $m$ and $n$ be integers such that $0 \leq m<n / 2$ and let $\sigma$ be as in (2.12). Let $A_{1}, A_{2}$ and $\rho_{3}$ be defined as in (2.6), (2.7), and (2.10) respectively. Let $\lambda$ be a real number in the interval $(1,2)$. Then as $n \rightarrow \infty$,

$$
\left(\frac{m^{1+s}(\log m)^{-t}}{n}\right)^{1 / \lambda} \underset{\lambda}{\ll} \mathrm{e}^{\sigma m} \ll \frac{m^{1+s}(\log m)^{-t}}{n},
$$

for $(6+2 s) / \sigma \leq m \leq n / 2$, where the implied constants depend only on $\lambda$ and the sequence $u$.

PROOF. Corollary 13 (ii) gives us that as $n \rightarrow \infty$ for $m \geq(6+2 s) / \sigma$,

$$
m^{1+s}(\log m)^{-t} \mathrm{e}^{-\sigma m \lambda} \underset{\lambda}{\ll} A_{1} \ll m^{1+s}(\log m)^{-t} \mathrm{e}^{-\sigma m} .
$$

By our choice of $\sigma$ in (2.12) we have $A_{1}=n$ and so we have immediately that

$$
m^{1+s}(\log m)^{-t} \mathrm{e}^{-\sigma m \lambda} \underset{\lambda}{\ll} n \ll m^{1+s}(\log m)^{-t} \mathrm{e}^{-\sigma m} .
$$

Rearranging the inequalities give the result. 
Finally, we present the following explicit estimates of the quantities $A_{2}$ and $\rho_{3}$ which combine to cover all $m$ in the range $0 \leq m \leq n / 2$.

LEMMA 17. Let $u$ be a strictly increasing sequence of positive integers satisfying Hypothesis $H$ and let $s$ and $t$ be as in Hypothesis $H$. Let $m$ and $n$ be integers such that $0 \leq m<n / 2$ and let $\sigma$ be as in (2.12). Let $A_{1}, A_{2}$ and $\rho_{3}$ be defined as in (2.6), (2.7), and (2.10). Let $\lambda$ be a real number in the interval $(1,2)$.

(i) Let $K \geq 1$ be a constant. For $0 \leq m \leq K / \sigma$, we have that as $n \rightarrow \infty$,

$$
\begin{aligned}
& \sigma^{-1} \asymp\left(n(\log n)^{t}\right)^{1 /(s+1)}, \quad A_{2} \widetilde{K} n^{(s+2) /(1+s)}(\log n)^{t /(1+s)}, \\
& \rho_{3} \underset{\kappa}{\asymp} n^{(s+3) /(s+1)}(\log n)^{t /(s+1)},
\end{aligned}
$$

where the implied constants depend only on $K$ and $u$.

(ii) For $(6+2 s) / \sigma \leq m \leq n / 2$, as $n \rightarrow \infty$, we have

$$
\frac{1}{2} m n<A_{2} \underset{\lambda}{\ll} m^{1+(1+s)(\lambda-1)} n^{2-\lambda}, \quad \rho_{3} \underset{\lambda}{\ll} m^{2+2(\lambda-1)(1+s)} n^{3-2 \lambda},
$$

where the implied constants depend only on $u$ and $\lambda$.

ProOF. (i) For $0 \leq m \leq K / \sigma$, we appeal to Corollary 13(i) and Lemma 14 to give

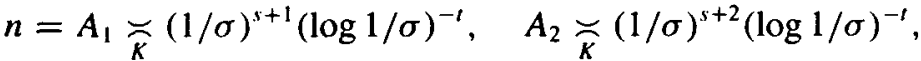

$$
\begin{aligned}
& \rho_{3} \asymp(1 / \sigma)^{s+3}(\log 1 / \sigma)^{-t},
\end{aligned}
$$

where the implied constants depend only on $K$ and $u$. Using the fact that $1 / \sigma \rightarrow \infty$ as $n \rightarrow \infty$ we can invert the expression for the magnitude of $A_{1}$ giving

$$
1 / \sigma \breve{K}^{\asymp} n^{1 /(s+1)}(\log n)^{t /(s+1)}
$$

from which we have

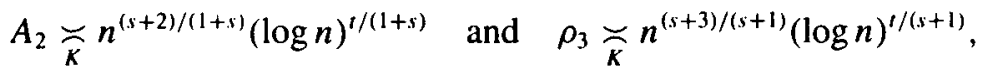

where the implied constants depend only on $K$ and $u$.

(ii) Combining (2.12), (8.7) and (8.8) gives the inequalities

$$
A_{2}=A_{1} \frac{A_{2}}{A_{1}}>A_{1} \frac{1}{2} \frac{\rho_{2}}{\rho_{1}}>\frac{1}{2} m n .
$$

Also Lemma 15 gives

$$
A_{2}=A_{1} \frac{A_{2}}{A_{1}}{ }_{\lambda} n m \mathrm{e}^{\sigma m(\lambda-1)} \quad \text { and } \quad \rho_{3}=A_{1} \frac{A_{2}}{A_{1}} \frac{\rho_{3}}{A_{2}} \underset{\lambda}{\ll} n\left(m \mathrm{e}^{\sigma m(\lambda-1)}\right)^{2} .
$$


Lemma 16 gives

$$
\mathrm{e}^{\sigma m} \ll \frac{m^{1+s}(\log m)^{-t}}{n} \ll \frac{m^{1+s}}{n},
$$

so that $A_{2} \ll n^{2-\lambda} m^{1+(1+s)(\lambda-1)}$ and $\rho_{3} \ll n^{3-2 \lambda} m^{2(1+(1+s)(\lambda-1))}$, from which the result follows.

\section{Estimation of supplementary integral}

We estimate the supplementary integral as given in (4.4).

LEMMA 18. Let $\lambda$ be a real number in the interval $(1,2)$. Let $u$ be a strictly increasing sequence of positive integers satisfying Hypothesis $H$ and Hypothesis $K$. Let $m$ and $n$ be integers such that $0 \leq m<n / 2$ and let $\sigma$ be as in (2.12) and let $A_{1}, A_{2}$ and $\rho_{3}$ be defined as in (2.6), (2.7), and (2.10). Let $\alpha_{0}$ be some number in the interval $(0,1 / 2)$ and let $S=\int_{\left\{\alpha: \alpha_{0} \leq|\alpha| \leq 1 / 2\right\}} \varphi(\alpha) \mathrm{e}(-\alpha n) d \alpha$, as in (4.4).

(i) Let $K \geq 1$ be a constant. There is a constant $n_{0}>0$ depending only on $u, \lambda$ and $K$ and there is a constant $R_{1}>0$ depending only on $K, \lambda$ and $u$ such that for $n>n_{0}$, if $0 \leq m \leq K / \sigma$, then $|S| \leq \exp \left(-R_{1} \min \left(\alpha_{0}^{2} \sigma^{-2}, 1\right) n^{s(2-\lambda) /(s+1)}\right)$.

(ii) There is a constant $n_{0}>0$ depending only on $\lambda$ and $u$ and there is a constant $R_{2}>0$ depending only on $\lambda$ and $u$ such that for $n>n_{0}$, if $(6+2 s) / \sigma \leq m \leq n / 2$, then $|S| \leq \exp \left(-R_{2} \min \left(\alpha_{0}^{2} m^{2}, 1\right) n^{\lambda} m^{2 s-2 \lambda s-\lambda}\right)$.

PROOF. From the above expression for the supplementary integral $S$ we have

$$
|S| \leq \sup _{\left\{\alpha: \alpha_{0} \leq|\alpha| \leq 1 / 2 \mid\right.}|\varphi(\alpha)| .
$$

From Lemma 8 we have an estimate of the size of $\varphi$ and hence an upper bound for $|S|$. This is the idea of the proof.

(i) Suppose $0 \leq m \leq K / \sigma$. Then, since $n \gg_{K} 1 / \sigma$ by Lemma 6 ,

$$
\sum_{m<u_{j} \leq n} \mathrm{e}^{-\sigma u_{j}}\left\|\alpha u_{j}\right\|^{2}>\sum_{K / \sigma<u_{j} \leq \lambda K / \sigma} \mathrm{e}^{-\sigma u_{j}}\left\|\alpha u_{j}\right\|^{2}>\mathrm{e}^{-\lambda K} \sum_{K / \sigma<u_{j} \leq \lambda K / \sigma}\left\|\alpha u_{j}\right\|^{2} .
$$

We consider two cases. Firstly, if $\alpha_{0}<|\alpha|<\sigma /(2 \lambda K)$, then using a lower bound on $U(\lambda K / \sigma)-U(K / \sigma)$ (similar to that provided in Lemma 3 for the case $\lambda=2$ ) and Hypothesis $\mathrm{H}$ we obtain

$$
\sum_{K / \sigma<u_{j} \leq \lambda K / \sigma}\left\|\alpha u_{j}\right\|^{2}=\sum_{K / \sigma<u_{j} \leq \lambda K / \sigma} \alpha^{2} u_{j}^{2} \gg_{K} \alpha_{0}^{2} \sigma^{-2-s}(\log 1 / \sigma)^{-t} .
$$


Lemma 5 (i) gives $1 / \sigma \gg n^{1 /(s+1)}$ and hence, since $1<\lambda<2$, the above expression is $\gg \alpha_{0}^{2} \sigma^{-2} n^{s(2-\lambda) /(s+1)}$.

Secondly, if $\sigma /(2 \lambda K)<|\alpha|<1 / 2$, then Hypothesis $K$ gives

$$
\sum_{K / \sigma<u_{j} \leq \lambda K / \sigma}\left\|\alpha u_{j}\right\|^{2} \underset{\lambda, K}{\gg}(1 / \sigma)^{s(2-\lambda)} \gg n^{s(2-\lambda) /(s+1)} .
$$

Hence from Lemma 8 we have the result (i).

(ii) Suppose $(6+2 s) / \sigma \leq m \leq n / 2$. Then

$$
\sum_{m<u_{j} \leq n} \mathrm{e}^{-\sigma u_{j}}\left\|\alpha u_{j}\right\|^{2}>\sum_{m<u_{j} \leq \lambda m} \mathrm{e}^{-\sigma u_{j}}\left\|\alpha u_{j}\right\|^{2}>\mathrm{e}^{-\lambda \sigma m} \sum_{m<u_{j} \leq \lambda m}\left\|\alpha u_{j}\right\|^{2} .
$$

From Lemma 16

$$
\mathrm{e}^{\sigma m} \ll \frac{m^{1+s}(\log m)^{-t}}{n} \ll \frac{m^{1+s}}{n}
$$

so that from $(9.1)$

$$
\sum_{m<u_{j} \leq n} \mathrm{e}^{-\sigma u_{j}}\left\|\alpha u_{j}\right\|^{2} \gg{ }_{\lambda}\left(n / m^{1+s}\right)^{\lambda} \sum_{m<u_{j} \leq \lambda m}\left\|\alpha u_{j}\right\|^{2} .
$$

We consider two cases. Firstly, if $\alpha_{0}<|\alpha|<1 /(2 \lambda m)$, then Hypothesis $\mathrm{H}$ and an argument along the lines of the proof of Lemma 3 give

$$
\sum_{m<u, \leq \lambda m}\left\|\alpha u_{j}\right\|^{2}=\sum_{m<u_{j} \leq \lambda m} \alpha^{2} u_{j}^{2} \gg \alpha_{\lambda}^{2} m^{2+s}(\log m)^{-1} \gg_{\lambda} \alpha_{0}^{2} m^{2} m^{s(2-\lambda)} .
$$

Secondly, if $1 /(2 \lambda m) \leq|\alpha|<1 / 2$ then Hypothesis $\mathrm{K}$ gives

$$
\sum_{m<u_{j} \leq \lambda m}\left\|\alpha u_{j}\right\|^{2} \gg m^{s(2-\lambda)} \text {. }
$$

In the light of (9.2), Lemma 8 and the remarks at the commencement of this proof, we have the result (ii).

\section{Proof of the main theorem}

Preliminaries to commencement of the proof Let $\delta>0$ be given such that $0<\delta<1$ (as in (4.1)). In order to prove Theorem 2 we must show that

$$
\begin{aligned}
\int_{-1 / 2}^{1 / 2} \varphi(\alpha) \mathrm{e}(-\alpha n) d \alpha= & \frac{1}{\sqrt{2 \pi A_{2}}}\left(1+O\left(n^{-s /(2 s+2)}(\log n)^{-t /(2 s+2)}\right)\right. \\
& \left.+O_{\delta}\left((m / n)^{16 / 33}\right)\right) .
\end{aligned}
$$


For any $\alpha_{0}$ such that $0<\alpha_{0}<1 / 2$ we have

$$
\int_{-1 / 2}^{1 / 2} \varphi(\alpha) \mathrm{e}(-\alpha n) d \alpha=M+S
$$

where, by Lemma 9

$$
M=\int_{-\alpha_{0}}^{\alpha_{0}} \varphi(\alpha) \mathrm{e}(-\alpha n) d \alpha=\frac{1}{\sqrt{2 \pi A_{2}}}\left(1+E_{1}\left(\alpha_{0}\right)\right)
$$

where $E_{1}\left(\alpha_{0}\right) \ll \rho_{3} / A_{2}^{3 / 2}+\mathrm{e}^{-2 \pi^{2} A_{2} \alpha_{0}^{2}}$, provided that the conditions (7.1)-(7.3) are satisfied, and Lemma 18 gives different upper bounds on

$$
S=\int_{\left\{\alpha: \alpha_{0} \leq|\alpha| \leq 1 / 2\right\}} \varphi(\alpha) \mathrm{e}(-\alpha n) d \alpha
$$

depending on whether or not $(6+2 s) / \sigma \leq m<n^{1-\delta}$.

We divide the main work of the proof into two parts, Part 1 corresponding to the case $1 \leq m \leq(6+2 s) / \sigma$ and Part 2 corresponding to the case $(6+2 s) / \sigma \leq m \leq n^{1-\delta}$.

Part 1 The case $0 \leq m \leq(6+2 s) / \sigma$.

For this case we shall show that for any $\lambda$ such that $1<\lambda<2$ we have

$$
\int_{-1 / 2}^{1 / 2} \varphi(\alpha) \mathrm{e}(-\alpha n) d \alpha=\frac{1}{\sqrt{2 \pi A_{2}}}\left(1+O_{\lambda}\left(n^{-s /(2 s+2)}(\log n)^{-t(2 s+2)}\right)\right) .
$$

We therefore now consider $\lambda$ such that $1<\lambda<2$, with $\lambda$ fixed throughout this part of the argument, and use this value of $\lambda$ in the various estimates.

In order to apply Lemma 9 we require that each of the conditions (7.1)-(7.3) hold. Thus what requires checking is whether

$$
\left(\frac{3}{10 \rho_{3}}\right)^{1 / 3}>2 \pi \alpha_{0}>\frac{1}{\sqrt{A_{2}}} \text { and } \frac{\sigma}{\log 3}>2 \pi \alpha_{0}
$$

It suffices to choose $\alpha_{0}$ satisfying (10.3) and we can simplify the choice for $\alpha_{0}$ by minimising the error terms $E_{1}$ and $E_{2}$ which are such that

$$
M=\frac{1}{\sqrt{2 \pi A_{2}}}\left(1+E_{1}\left(\alpha_{0}\right)\right) \quad \text { and } \quad S=\frac{E_{2}\left(\alpha_{0}\right)}{\sqrt{2 \pi A_{2}}} .
$$

Lemma 9 gives

$$
E_{1}\left(\alpha_{0}\right) \ll \frac{\rho_{3}}{A_{2}^{3 / 2}}+\mathrm{e}^{-2 \pi^{2} A_{2} \alpha_{0}^{2}}
$$


Lemma 18 (i) gives

$$
|S| \leq \exp \left(-R_{1} \min \left(\alpha_{0}^{2} \sigma^{-2}, 1\right) n^{s(2-\lambda) /(s+1)}\right),
$$

where $R_{1}=R_{1}(\lambda)$ depends only on $\lambda$ and $u$. It is clear that making $\alpha_{0}$ as large as possible will minimise $E_{1}$ and $E_{2}$.

Now from Lemma 17(i) (with the choice $K=6+2 s$ )

$$
\begin{aligned}
A_{2} & \asymp n^{(2+s) /(1+s)}(\log n)^{t /(1+s)}, \\
\rho_{3} & \asymp n^{(s+3) /(s+1)}(\log n)^{t /(s+1)}, \\
\rho_{3} / A_{2}^{3 / 2} & \asymp n^{-s /(2 s+2)}(\log n)^{-t /(2 s+2)},
\end{aligned}
$$

where the implied constants depend only on $u$.

Thus

$$
\begin{aligned}
\frac{1}{2 \pi}\left(\frac{3}{10 \rho_{3}}\right)^{1 / 3} & \gg n^{-(1+s) /(3(3+s))}(\log n)^{-t /(3(1+s))}, \\
\sigma /(2 \pi \log 3) & \gg n^{-1 /(1+s)}(\log n)^{-t /(1+s)} \text { and } \\
\frac{1}{2 \pi \sqrt{A_{2}}} & \ll n^{-(1+s) /(2(2+s))}(\log n)^{-t /(2(1+s)) .}
\end{aligned}
$$

Hence there is a positive constant $K_{4}$ (depending only on $u$ ) such that the choice

$$
\alpha_{0}=K_{4} n^{-1 /(1+s)}(\log n)^{-t /(1+s)}
$$

for $\alpha_{0}$ is consistent with (10.9) and (10.3).

In the light of (10.8) and the above choice for $\alpha_{0}$ in (10.10) we have from (10.5) that $E_{1}\left(\alpha_{0}\right) \ll n^{-s /(2 s+2)}(\log n)^{-t /(2 s+2)}$. Using the lower bound for $\sigma^{-1}$ from Lemma 17 (i) and the choice for $\alpha_{0}$ in (10.10) we have from (10.4), (10.6) and the the fact that $2-\lambda>0$, we obtain $E_{2}\left(\alpha_{0}\right) \ll n^{-1} \ll n^{-s /(2 s+2)}(\log n)^{-1 /(2 s+2)}$, and the conclusion (10.2) follows.

Part 2 This is the case where $n^{1-\delta} \geq m \geq(6+2 s) / \sigma$.

For this case we shall show that for any $\lambda$ such that $1<\lambda \leq 1+\delta / 33$ we have

$$
\int_{-1 / 2}^{1 / 2} \varphi(\alpha) \mathrm{e}(-\alpha n) d \alpha=\frac{1}{\sqrt{2 \pi A_{2}}}\left(1+O_{\lambda}\left((m / n)^{16 / 33}\right)\right) .
$$

(The reason for the upper bound $1+\delta / 33$ will become clear later in the argument). We therefore take $\lambda$ such that $1<\lambda \leq 1+\delta / 33$, fixed throughout this part of the argument, and we note that this implies

$$
\delta \geq 33(\lambda-1) .
$$


As in Part 1, in order to apply Lemma 9 we require that each of the conditions (7.1)-(7.3) hold. Automatically condition (7.3) holds. All that remains to be checked is whether

$$
\left(\frac{3}{10 \rho_{3}}\right)^{1 / 3}>2 \pi \alpha_{0}>\frac{1}{\sqrt{A_{2}}} .
$$

Thus it suffices to choose $\alpha_{0}$ satisfying (10.13). It is clear from the bound for $E_{1}\left(\alpha_{0}\right)$ in Lemma 9 and the bound for $|S|$ in Lemma 18 (ii) that making $\alpha_{0}$ as large as possible will minimise $E_{1}$ and $E_{2}$ from (10.4).

From Lemma 17 (ii) we obtain

$$
\frac{1}{2 \pi}\left(\frac{3}{10 \rho_{3}}\right)^{1 / 3} \gg m^{-2(1+(\lambda-1)(1+s)) / 3} n^{-7 / 3+2 \lambda}
$$

and

$$
\frac{1}{2 \pi \sqrt{A_{2}}}<\frac{1}{\sqrt{2} \pi} n^{-1 / 2} m^{-1 / 2}
$$

Hence there is a constant $K_{5}>0$ depending only on $\lambda$ and $u$ such that the choice for $\alpha_{0}$, namely

$$
\alpha_{0}=K_{5} m^{-2(1+(\lambda-1)(1+s)) / 3} n^{-7 / 3+2 \lambda},
$$

is consistent with (10.13). It follows from the bound on $\rho_{3} / A_{2}^{3 / 2}$ in Lemma 17 (ii) and the choice for $\alpha_{0}$ in (10.14) that the bound for $E_{1}\left(\alpha_{0}\right)$ in Lemma 9 becomes

$$
E_{1}\left(\alpha_{0}\right) \ll \frac{m^{(1+(1+s)(\lambda-1) / 2}}{n^{\lambda / 2}}+\exp \left(-2 \pi^{2} \frac{1}{2} m n\left(m^{-2(1+(\lambda-1)(1+s)) / 3} n^{-7 / 3+2 \lambda}\right)^{2}\right) .
$$

Since the exponent in the exponential function exp above is

we have

$$
\asymp(n / m)^{1 / 3}\left(n / m^{(1+s) / 3}\right)^{4 \lambda-4} \gg \frac{n^{1 / 3}}{m^{1 / 3}}
$$

$$
E_{1}\left(\alpha_{0}\right) \underset{\lambda}{\ll} \frac{m^{(1+(1+s)(\lambda-1) / 2}}{n^{\lambda / 2}} .
$$

Also using (10.4) and the bound for $S$ in Lemma 18 (ii) and then substituting (10.14) we obtain

$$
E_{2}\left(\alpha_{0}\right) \underset{\lambda}{\ll} m^{1 / 2+(1+\cdots)(i-1) / 2} n^{(2-i) / 2} \exp \left(-R_{4} m^{-1 / 3-(7+1(1))(\lambda-1) / 3} n^{-14 / 3+5 \lambda}\right)
$$


for some positive constant $R_{4}$ depending only on $\lambda$ and $u$. Because

$$
m^{-1 / 3-(7+10 s)(\lambda-1) / 3} n^{-14 / 3+5 \lambda}>n^{\delta / 3-32(\lambda-1) / 3} \text { for } m<n^{1-\delta}
$$

having $\delta \geq 33(\lambda-1)$ ensures that

$$
\exp \left(-R_{4} m^{-1 / 3-(7+10 s)(\lambda-1) / 3} n^{-14 / 3+5 \lambda}\right) \ll n^{-1}
$$

and hence that

$$
E_{2}\left(\alpha_{0}\right) \underset{\lambda}{\ll} \frac{m^{1 / 2+(1+s)(\lambda-1) / 2}}{n^{\lambda / 2}} .
$$

We can simplify the bound on the error terms $E_{1}$ and $E_{2}$ by writing

$$
\frac{m^{1 / 2+(1+s)(\lambda-1) / 2}}{n^{\lambda / 2}}=\left(\frac{m}{n}\right)^{16 / 33} \times \frac{m^{1 / 66+(1+s)(\lambda-1) / 2}}{n^{1 / 66+(\lambda-1) / 2}}
$$

Using the condition $m \leq n^{1-\delta}$ and that $s \leq 1$, it can be checked that

$$
\frac{m^{1 / 66+(1+s)(\lambda-1) / 2}}{n^{1 / 66+(\lambda-1) / 2}}<n^{-\delta / 66+(\lambda-1) / 2-\delta(\lambda-1) / 2},
$$

and this last expression will be less than unity provided that the exponent of $n$ is negative. It is sufficient that (10.12) be satisfied. Thus the bounds on the error terms $E_{1}$ and $E_{2}$ are $O_{\delta}\left((m / n)^{16 / 33}\right)$ as required in (10.11).

Completion of the proof If $0 \leq m \leq(6+2 s) / \sigma$, we use a particular $\lambda$ in Part 1 , say $\lambda=3 / 2$, giving

$$
\int_{-1 / 2}^{1 / 2} \varphi(\alpha) \mathrm{e}(-\alpha n) d \alpha=\frac{1}{\sqrt{2 \pi A_{2}}}\left(1+O\left(n^{-s /(2 s+2)}(\log n)^{-t /(2 s+2)}\right)\right) .
$$

where the implied constants are absolute. We note that in the proof of Lemma 14 the choice $\lambda=3 / 2$ was made, but could equally have been any other specified $\lambda$.

If $m \geq(6+2 s) / \sigma$, we take $\lambda=1+\delta / 33$ in Part 2, giving

$$
\int_{-1 / 2}^{1 / 2} \varphi(\alpha) \mathrm{e}(-\alpha n) d \alpha=\frac{1}{\sqrt{2 \pi A_{2}}}\left(1+O_{\delta}\left((m / n)^{16 / 33}\right)\right)
$$

Thus in all cases we obtain (10.1), as required. This completes the proof of the main theorem of this paper. 


\section{Discussion}

Modifications of main theorem We can modify the main theorem to accomodate 'small' $m$ more precisely. We note that for $m=o\left(n^{1 /(1+s)}(\log n)^{t /(1+s)}\right)$ we have by Lemma 17 (i) that $m<(6+2 s) / \sigma$. Hence Part 1 of the proof of Theorem 2 is relevant and we have as a corollary of Theorem 2 the following theorem.

THEOREM 19. Let $u$ be a strictly increasing sequence of positive integers satisfying Hypotheses $\mathrm{H}$ and $\mathrm{K}$. Let $q_{u}(m, n)$ be as above and let $\sigma$ and $A_{2}$ be as in be as in (2.12) and (2.7). Then as $n \rightarrow \infty$,

$$
\begin{aligned}
q_{u}(m, n)= & \mathrm{e}^{\sigma n} \prod_{m<u, \leq n}\left(1+\mathrm{e}^{-\sigma u_{j}}\right) \frac{1}{\sqrt{2 \pi A_{2}}} \\
& \times\left(1+O_{u}\left(n^{-s /(2(s+1))}(\log n)^{-t /(2 s+2)}\right)\right),
\end{aligned}
$$

for $m=o\left(n^{1 /(1+s)}(\log n)^{t /(1+s)}\right)$.

As an application, this theorem can be used for more refined results on $q_{u}(m, n)$. When $u=\left(j^{k}\right)$ an asymptotic estimate explicit in $m$ and $n$ can be obtained. This will be done in a subsequent paper. Applications of this theorem extend to the sequence of prime numbers as well as a wide range of uniformly distributed sequences and polynomial sequences.

Hypotheses on sequence The hypotheses of Roth and Szekeres are sufficient to prove a theorem like Theorem 2 certainly when $m=0$ and without much modification, when $m$ is not 'too large'. However, it appeared difficult to provide an estimate of $q_{u}(m, n)$ for $m$ 'large' under Hypotheses (RS1) and (RS2) of Roth and Szekeres because the asymptotic estimation of the cumulants when $\mathrm{m}$ is 'large' required a knowledge of the behaviour of $u_{j}$ more detailed than that deducible from Hypothesis (RS1). This was the reason for the tightening of (RS1).

Although not pursued in this paper, there is the possibility of weakening Hypotheses $\mathrm{H}$ and $\mathrm{K}$ by introducing the concept of regularly varying functions. The function $C_{0} x^{s}(\log x)^{-t}$ is regularly varying and this suggests that it may be possible replace Hypothesis $\mathrm{H}$ by the condition that $U(x)$ is regularly varying.

\section{References}

[1] R. Ash, Real analysis and probability (Academic Press, New York, 1972).

[2] J. W. S. Cassels, 'On the representation of integers as the sums of distinct summands taken from a fixed set', Acta Sci. Math. Szeged 21 (1960), 111-124. 
[3] P. Erdôs, J. Nicolas and M. Szalay, Partitions into parts which are unequal and largeLecture Notes in Math. 1380 (Springer, Berlin, 1989) pp. 19-30.

[4] K. Fergusson, 'Partitions into $k$-th powers', preprint.

[5] - Partitions into unequal large parts (Ph.D. Thesis, The University of Adelaide, Adelaide, South Australia, 1996).

[6] G. Freiman, 'On extremal additive problems of Paul Erdôs', Ars Combin. 26B (1988), 93-114.

[7] G. Freiman and J. Pitman, 'Partitions into distinct large parts', J. Austral. Math. Soc. 57 (1994), $386-416$.

[8] B. Gnedenko and A. Kolmogorov, Limit distributions for sums of independent random variables (Addison-Wesley, Cambridge, Massachusetts, 1954).

[9] G. Hardy and S. Ramanujan, 'Asymptotic formulae in combinatory analysis', Proc. London Math. Soc. 17 (1918), 75-115.

[10] L. Hua, 'On the number of partitions of a number into unequal parts', Trans. Amer. Math. Soc. 51 (1942), 194-201.

[11] A. E. Ingham, The distribution of prime numbers, Cambridge Tracts in Mathematics 30 (Cambridge University Press, London, 1932).

[12] _- 'A Tauberian theorem for partitions', Ann. of Math. (2) 42 (1941), 1075-1090.

[13] A. Postnikov, Introduction to analytic number theory, Translations of mathematical monographs 68 (American Mathematical Society, Providence, RI, 1988).

[14] K. Roth and G. Szekeres, 'Some asymptotic formulae in the theory of partitions', Quart. J. Math. (Oxford) (2) 5 (1954), 241-259.

[15] M. Spivak, Calculus, 2nd edition (Publish or Perish, Berkeley, CA, 1980).

[16] J. Uspensky, 'Asymptotic expressions of numerical functions occurring in problems of partition of numbers', Bull. Acad. Sci. de Russie 14 (1920), 199-218.

\section{Blackdown way}

Karrinyup WA 6018

Australia

e-mail: kevinjohnfergusson@hotmail.com 